\title{
Prototype Development of Paper Based Sensor for Ammonia Detection in Water
}

\author{
Muhammad Hambali Habibullah, Mastura Shafinaz Zainal Abidin* \\ and Shaharin Fadzli Abd Rahman
}

School of Electrical Engineering, Universiti Teknologi Malaysia, 81310 UTM Johor Bahru, Johor, Malaysia.

"Corresponding author: m-shafinaz@utm.my

\begin{abstract}
The presence of ammonia in water supply can be harmful to human health. The ammonia is colorless, thus its presence not easily detected. Therefore, a suitable sensor for this detection is necessary. The sensor device developed in this study was utilizing bio-environmental friendly materials such as paper and graphite. Several combination of paper and graphite type were used to evaluate the sensitivity and selectivity of sensor. A suitable device pattern was defined as the sensing area for detecting the presence of ammonia in water. The device was connected with a constant voltage to generate the current flow between electrodes made of graphite and the liquid sample was tested by drop it onto the sensing area. As the result, the significant current changes could be detected with presence of ammonia in deionized (DI) water at different concentration level. A zinc oxide layer was proposed to be added at the sensing area for sensing enhancement. Based on the sensing response, the presence of zinc oxide demonstrated improvement on device sensitivity by displaying faster response characteristics. Thus, the combination of graphite and zinc oxide at sensing area is recommended where it able to better distinguish the level of ammonia concentration compared to those sensing area without zinc oxide. Besides low in cost, this biodegradable ammonia sensor prototype is considered safe and easy to be used by normal consumer for monitoring water quality.
\end{abstract}

Keywords: paper, graphite, zinc oxide, ammonia.

Article History: received 7 May 2018; accepted 15 August 2018; published 29 August 2018.

(C) 2018 Penerbit UTM Press. All rights reserved

\section{INTRODUCTION}

The water pollution is an issue to be concerned as it endangered civilian health. As enforced by National Water Quality Standard, the preservation and protection towards the water quality is a must to be monitored [1]. Many cases were reported regarding water pollution due to presence of numerous operators such as ammonia, bacteria and protozoans which might cause death [2]. Recently in Malaysia, the water pollution due to ammonia occurred at Sungai Cheras and Sungai Johor. This hazard was difficult to be detected with naked eye and the local people basically did not aware of the presence of ammonia in water. Hence, water supplies at this area were immediately closed to consumer for safety reason [3].

This study is proposed to develop a prototype sensor that suitable for everyone usage in detecting the presence of ammonia in water supplies, by offering the features of economical-based device and environmental friendlybased resource material. In addition of low cost approach benefit [4], the prototype developed also applying simple operation to monitor the level of ammonia concentration in water. The level of ammonia concentration can be detected by measuring the changes of current at specific applied voltage through the device [5].

Different types of paper and graphite were used as substrate and an electrode respectively in developing the sensor prototype [6]. Paper was chosen at different thickness and porosity type [7]. Graphite also was chosen based on the variation mixture of clay and graphite which represented by pencil grade such as $2 \mathrm{~B}$ and $6 \mathrm{~B}$. Besides working as electrode, the graphite also acted as active sensing area of device in this study. Different sensor area patterns were designed for optimization in order to clarify the sensitivity of sensor [8]. The effect of additional zinc oxide layer on graphite also been characterized [9]. Time response will be evaluated to determine the device sensitivity for detecting the concentration of ammonia presence in water.

\section{METHODOLOGY}

The processes involved in this study could be divided into two phases. Firstly, the study was aiming to clarify the sensing behavior according to base material source. The suitability of materials used were identified based on consistency in current changes response observed. The design pattern of the sensor also supposed to give effects towards sensitivity of the sensor [10].

Secondly, the study was extended to observe the effect of zinc oxide in the fabricated target device. The characteristics of zinc oxide with graphite acted as sensing element were observed. The time response and sensing rate of the fabricated sensor to detect different concentration level of ammonia were used as indicator. The general 
flowchart for this study is shown in Figure 1.

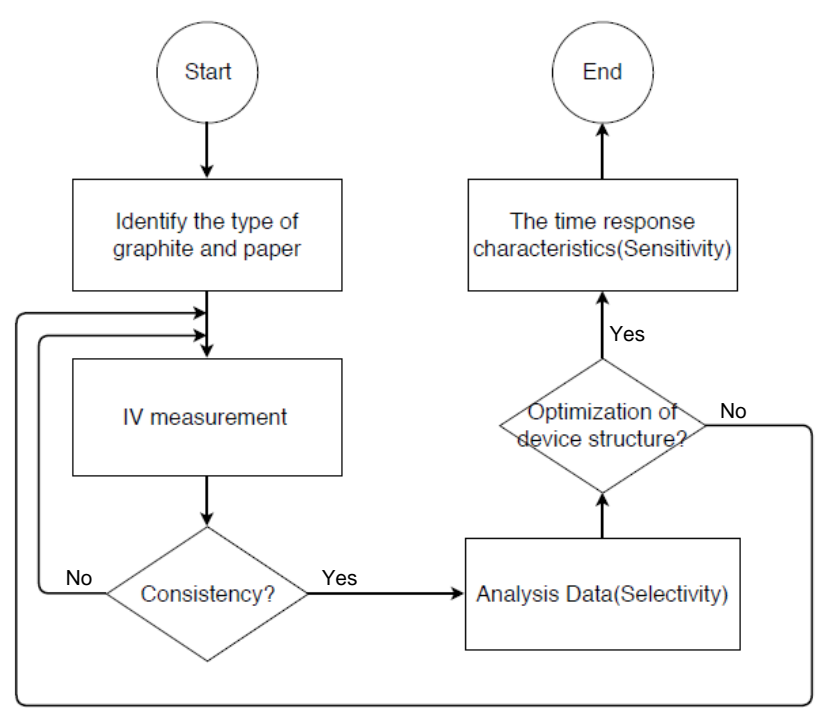

Figure 1. Overall process flowchart

For the first part, the base materials used were paper and graphite. The pencil grade of $2 \mathrm{~B}$ and $6 \mathrm{~B}$ were chosen as the source of different type of graphite because they indicated different percentage ratio between graphite and clay [11]. The Faber Castell pencil was chosen to be used in this study due to its quality and availability in different degree of hardness. The paper types being selected based on thickness as described by their weight in grams per square meter (gsm), and surface roughness specifications, as summarized in Table 1 .

Table 1. Specification of paper substrate

\begin{tabular}{|l|c|c|}
\hline \multicolumn{1}{|c|}{ Type } & gsm & Surface \\
\hline Cardboard paper & 240 & Smooth \\
\hline Whatman filter paper & 80 & Rough \\
\hline A4 printing & 80 & Smooth \\
\hline
\end{tabular}

As illustrated in Figure 2, three different design patterns would be transferred on paper substrate were attempted in this study. The patterns were designed to act as a sensing element of the sensor [12].

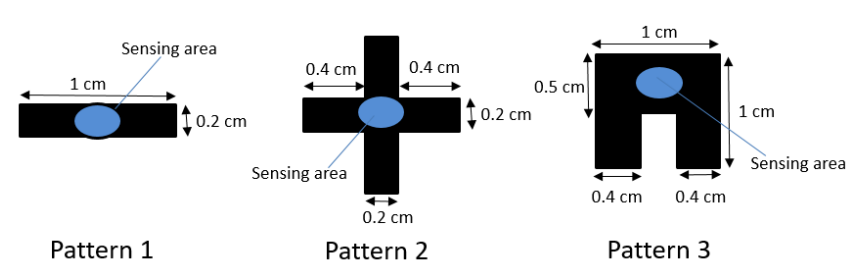

Figure 2. Design pattern of sensing area of proposed device.

Keithley 2000 was used to measure the changes of current indicated by the sensor upon samples' detection with the applied constant voltage of $10 \mathrm{~V}$ [13]. Semiconductor Device Analyzer tool also was used to measure the time response of sensing for each device structures investigated.

\subsection{Preparation of graphite on paper.}

The sensor was fabricated by simply drawing the graphite traces using pencils on paper. Graphite acted as an electrode as well as the sensing area. The detailed steps are listed as follows:

1. Cut the piece of paper sample into square shape with dimension of $(2 \mathrm{~cm} \times 2 \mathrm{~cm})$ by using a pair of scissors.

2. Draw the design pattern as indicated in Figure 2 respectively on the paper using pencil as source of graphite.

3. Drop a sample $(0.1 \mu \mathrm{L})$ of deionized water (DI) and ammonia mixture onto the platform of the sensing area.

4. Connect the graphite electrode with probe to Keithley 2000 to measure the related parameters such currentvoltage (I-V) response.

5. Repeat the step (1) until (4), using various combination of paper types and pencil grades to observe their effects on the selectivity behavior and trends.

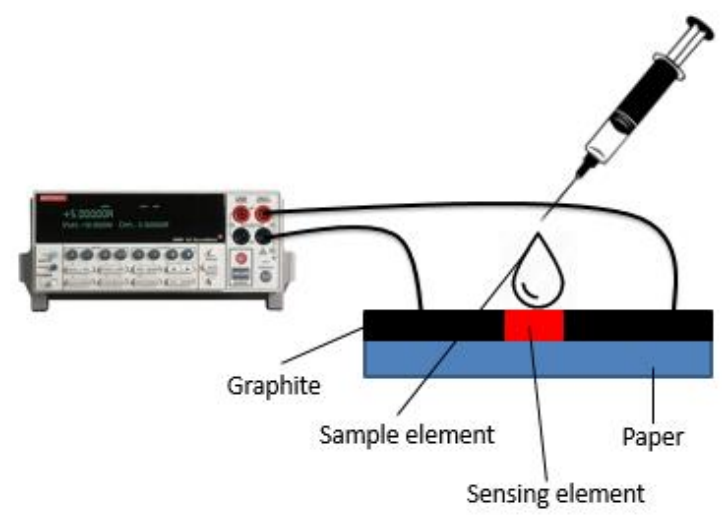

Figure 3. The configuration setup of I-V response measurement

\subsection{Preparation of zinc oxide layer at sensing area on paper}

To further observe the effect of additional zinc oxide layer presence, a set of device fabrication using combination of $6 \mathrm{~B}$ grade of graphite and A4 paper with design pattern 3 was repeated.

There were three different combinations of material structure at sensing region were investigated as shown in Figure 4. The examples of fabricated samples corresponded to each structures are shown in Figure 5.
Sensor 1

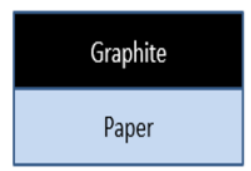

Sensing region of fabricate graphite on paper

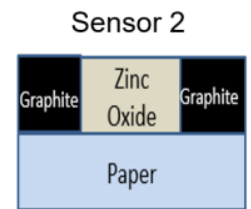

Sensing region of fabricate zinc oxide on paper paper

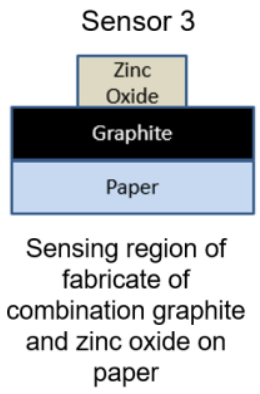

Figure 4. Material structure of sensing region. 


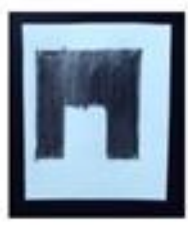

(a)

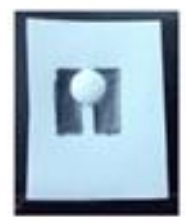

(b)

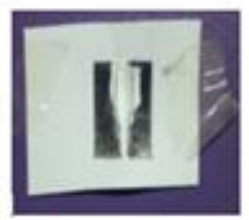

(c)
Figure 5. Sample of sensor fabricated on paper according to material structure of (a) Sensor 1. (b) Sensor 2. (c) Sensor 3.

Once the design pattern 3 was drawn on paper, zinc oxide was deposited on Sensor 2 and 3 structure. A $10 \mu \mathrm{L}$ aqueous suspension of $\mathrm{ZnO}$ crystals (Sigma Aldrich $<100$ $\mathrm{nm}$ particle size, $20 \%$ in $\mathrm{H}_{2} \mathrm{O}$ ) was placed at defined area, followed by $120^{\circ} \mathrm{C}$ heating for 2 minutes on a thermal hot plate in order to remove surplus solution. Next, the I-V characteristic and time response of each sensor will be measured by locating a droplet of prescribed ammonia concentration on the defined sensing area. The changes in current reading could be observed upon detection of different concentration level of ammonia tested. The measurement setup using Semiconductor Device Analyzer was applied as shown in Figure 6.

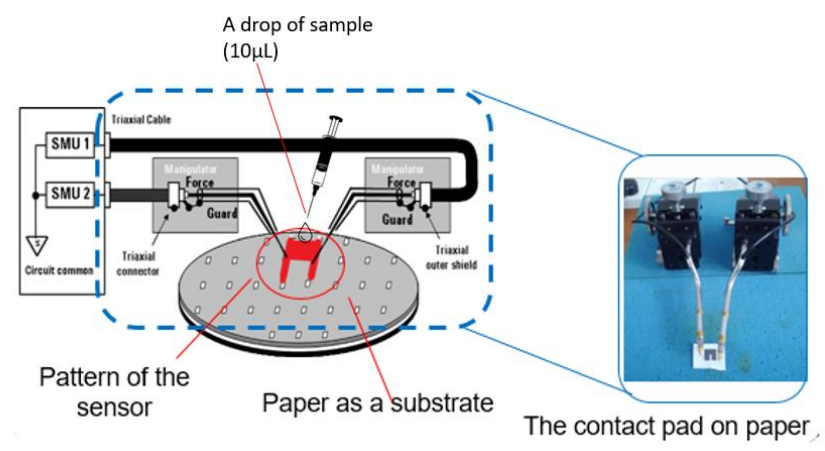

Figure 6. Configuration setup Using Semiconductor Device Analyzer.

\section{RESULT AND ANALYSIS}

\subsection{Effects of elements paper and graphite type.}

In this part, series of experiments were conducted to verify the selectivity and sensitivity of the sensor by fabricating several combination of paper types and graphite grades using design pattern 1, 2 and 3 as described in Figure 2. The relationship between current changes upon detection of samples at several conditions were studied. The current reading at dry condition was set as initial reference. Then, the current changes would be observed when DI water or mixture of ammonia and DI water were detected. In this study, the sample of ammonia and DI water mixture has been prepared by adding a $50 \mathrm{ml}$ ammonia with $50 \mathrm{ml}$ DI water. The concentration level of ammonia presence of samples were indicated in percentage based on volume ratio between ammonia and DI water mixture. Thus, $0 \%$ and $50 \%$ were used as indicator of ammonia level for this particular experiment.
The I-V response of different condition detected for device fabricated on $\mathrm{A} 4$ printing paper using $2 \mathrm{~B}$ and $6 \mathrm{~B}$ grade pencil with design pattern 1,2 , and 3 are shown in Figure 7, 8 and 9 respectively. Based on the graphs, Pattern 3 suggested more significant current changes upon detection of samples' condition compared to Pattern 1 and 2. Therefore, Pattern 3 had been chosen to be further investigated in the subsequent part.

The summary of average current changes from three readings corresponded to DI water and ammonia samples' presence, for all combination base material and design pattern in this study were tabulated in Table 2. Based on these data, the combination between A4 $80 \mathrm{gsm}$ printing paper and 6B grade Faber Castell pencil gave the best response in this study as the measurement results obtained using this material combination indicated the most consistent and gave the highest current changes compared to others. Therefore, the presence of samples could be significantly detected.

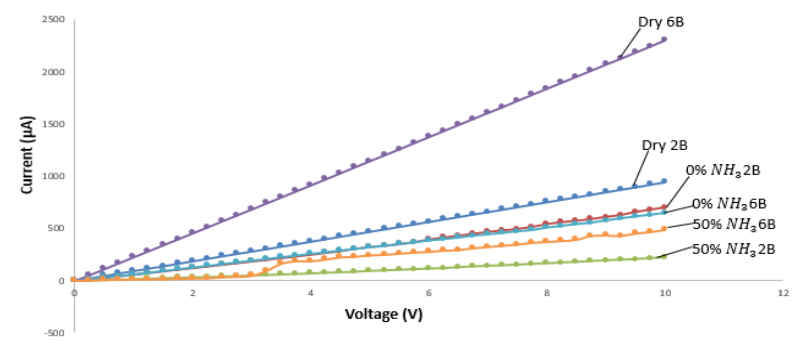

Figure 7. I-V response of device at different samples' conditions on Pattern 1

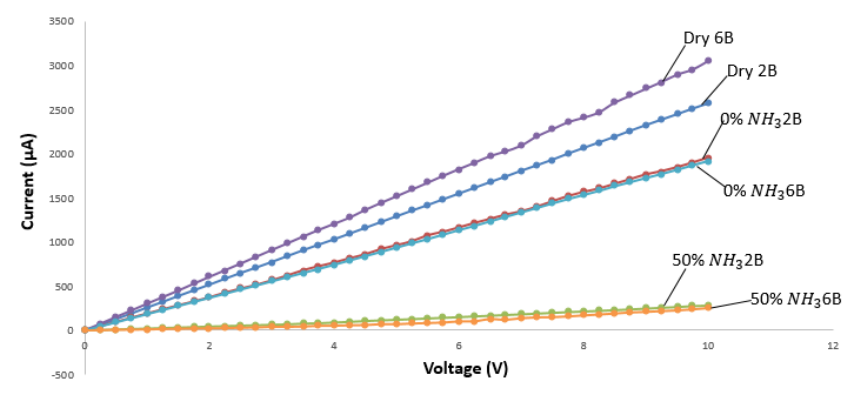

Figure 8. I-V response of device at different samples' conditions on Pattern 2

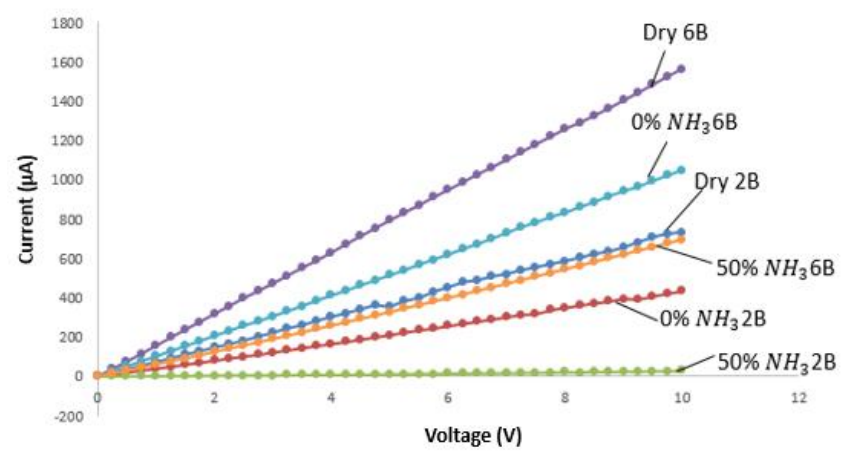

Figure 9. I-V response of device at different samples' conditions on Pattern 3 
Table 2. Average Current Response for DI Water Sample on pattern 3

\begin{tabular}{|l|c|c|c|}
\hline \multicolumn{2}{|c|}{ Design pattern 3 } & \multicolumn{2}{c|}{ Current changes $(\boldsymbol{\mu A})$} \\
\hline $\begin{array}{c}\text { Paper } \\
\text { Type }\end{array}$ & Graphite & DI water & Ammonia \\
\hline Cardboard & 2B & 75 & 9 \\
\cline { 2 - 4 } & 6B & 92 & 148 \\
\hline $\begin{array}{l}\text { Whatman } \\
\text { filter }\end{array}$ & 2B & 433 & 18 \\
\cline { 2 - 4 } $\begin{array}{l}\text { A4 } \\
\text { printing }\end{array}$ & 6B & 620 & 254 \\
\cline { 2 - 4 } & 2B & 244 & 24 \\
\hline
\end{tabular}

\subsection{Effects of elements paper and graphite type.}

Figure 10 shows the measured I-V characteristics for paper-based sensor with Pattern 3 using different material structure at sensing region upon detection of different ammonia's concentration levels. It seems that there was easier to differentiate the level of ammonia concentration based on Sensor 1 and 3 as the differences in current changes were clearly observed with significant trend.

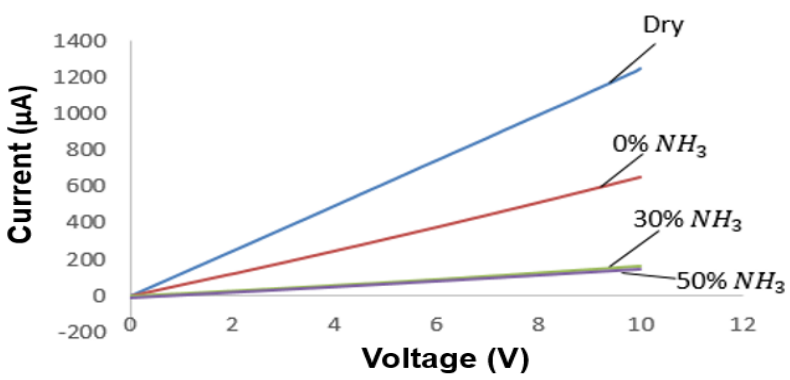

(a)

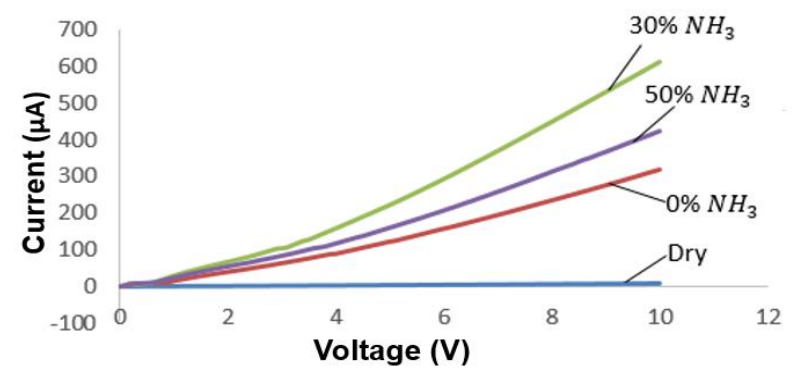

(b)

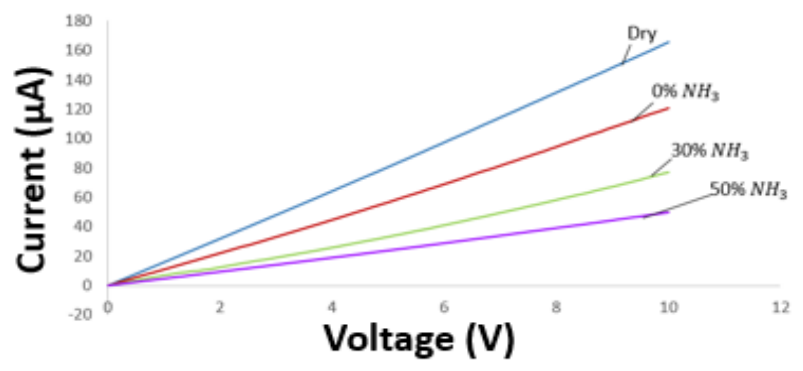

(c)

Figure 10. I-V response towards different ammonia's concentration level of paper-based sensor with Pattern 3 using different material structure at sensing region. (a) Sensor 1. (b) Sensor 2. (c) Sensor 3.

In order to observe the effect of additional zinc oxide layer presence, the sensing time response measurement was performed for all Sensor 1, 2 and 3. A constant $10 \mathrm{~V}$ was applied and a droplet of ammonia samples with $0 \%$, $30 \%$ and $50 \%$ concentration was placed at the defined sensing region at $\mathrm{t}=4 \mathrm{~s}$. Figure 11 shows the sensing response measured for respective devices. Generally, it can be seen that the additional layer of zinc oxide in sensing region had improve the sensitivity of sensor as the time taken to detect the presence of ammonia sample was shorter compared to graphite alone. The capability to sense the ammonia sample was observed for all three sensors. However, the selectivity performances were likely poor for Sensor 1 and 2, compared with Sensor 3. Thus, it could be concluded from this study that the paper based structure of Sensor 3 was the most suitable to be implemented as it capable to behave in suitable trends and properties of sensor in detecting the ammonia presence in water.

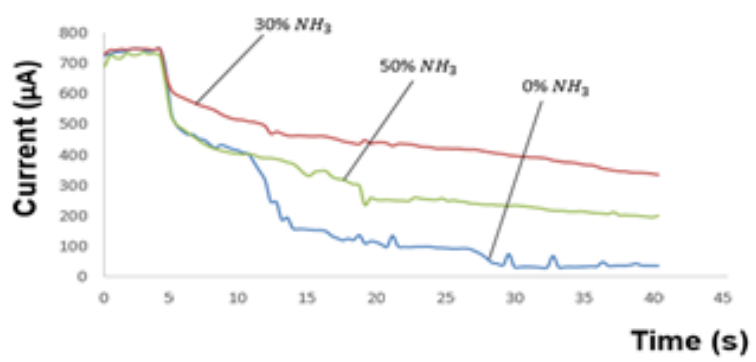

(a)

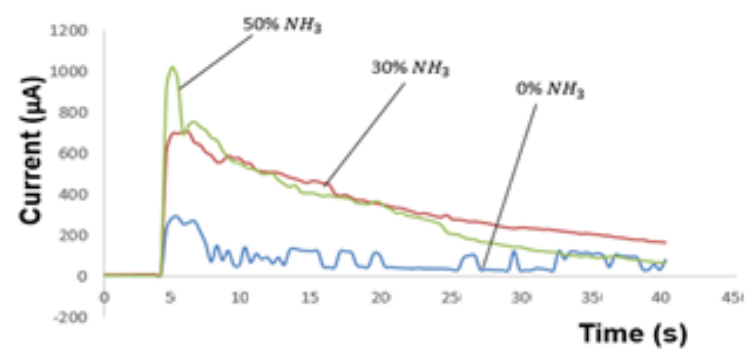

(b)

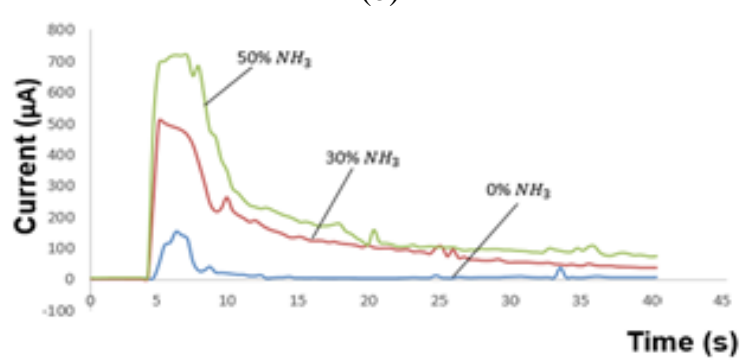

(c)

Figure 11. Sensing time response towards different ammonia's concentration level of paper-based sensor with Pattern 3 using different material structure at sensing region. (a) Sensor 1. (b) Sensor 2. (c) Sensor 3.

\section{CONCLUSION}

The fabrication of ammonia sensor using bioenvironment friendly materials such paper and graphite was implemented in this study. It offers benefits in term of easy implementation and low development cost. The suitable sensor design was studied based on the consistency in current response upon detection. Based on experiment conducted, the combination of $6 \mathrm{~B}$ pencil as source of graphite and A4 printing paper as the substrate gave the best response and sensing region's design pattern 
3 established better sensitivity behavior compared to Pattern 1 and 2 for detecting the presence of ammonia in DI water. With presence of zinc oxide, the sensing performance of the proposed sensor device could be improved as the time taken to detect the ammonia sample was shorter compared to zinc oxide absence. The higher mobility of ions makes the zinc oxide more sensitive than graphite alone. Therefore, it is expected that this ammonia sensor prototype would be applicable in monitoring the water quality by further optimize its details of development and preparation processes.

\section{ACKNOWLEDGMENT}

The authors would like to acknowledge the financial supports from Universiti Teknologi Malaysia and Ministry of Higher Education through project GUP- Tier 1 (13J78) and FRGS (4F926) grants. The authors also wish to express their appreciation to the authorities of the MicroNano Mechatronics Laboratory, and School of Electrical Engineering for all kinds of technical supports and FYP fund to make the project successful.

\section{REFERENCES}

[1] International Organization for Standardization. Water quality-determination of ammonium. Geneva, 1984, 1986 (ISO5664:1984; ISO6778:1984; ISO71501:1984; ISO7150-2:1986.

[2] Summary review of health effects associated with ammonia. Washington, DC, US Environmental Protection Agency, 1989 (EPA/600/8-89/052F).

[3] H. H. Dieter and R. Moller, "The drinking water regulations, introduction and explainations," Ammonium, pp. 362-368, 1991.

[4] Liana, D. D., Raguse, B., Justin Gooding, J., \& Chow, E. (2012). Recent advances in paper-based sensors. Sensors (Switzerland), 12(9), 11505-11526. https://doi.org/10.3390/s120911505

[5] Mirica, K. A., Weis, J. G., Schnorr, J. M., Esser, B., \& Swager, T. M. (2012). Mechanical drawing of gas sensors on paper. Angewandte Chemie International Edition, 51(43), 10740-10745. https://doi.org/10.1002/anie.201206069
[6] Kurra, N., \& Kulkarni, G. U. (2013). Pencil-onpaper: electronic devices. Lab on a Chip, 13(15), 2866. http://doi.org/10.1039/c3lc50406a.

[7] Martinez, A. W., Phillips, S. T., Butte, M. J., \& Whitesides, G. M. (2007). Patterned paper as a platform for inexpensive, low-volume, portable bioassays. Angewandte Chemie - International Edition, 46(8), 1318-1320. https://doi.org/10.1002/anie.200603817

[8] Carrilho, E., Martinez, B. A. W., \& B, G. M. W. (2009). Wax Printing - a Simple Micropatterning Process for Paper-based Microfluidics, 81(16), 1-5. http://doi.org/10.1021/ac901071p.

[9] Niarchos, G., Dubourg, G., Afroudakis, G., Georgopoulos, M., Tsouti, V., Makarona, E., Tsamis, C. (2017). Humidity Sensing Properties of Paper Substrates and Their Passivation with $\mathrm{ZnO}$ Nanoparticles for Sensor Applications. Sensors, 17(3), 516. https://doi.org/10.3390/s17030516.

[10] Tobjörk, D., \& Österbacka, R. (2011). Paper electronics. Advanced Materials, 23(17), 19351961. http://doi.org/10.1002/adma.201004692.

[11] Siegel, A. C., Phillips, S. T., Dickey, M. D., Lu, N., Suo, Z., \& Whitesides, G. M. (2010). Foldable printed circuit boards on paper substrates. Advanced Functional Materials, 20(1), 28-35. http://doi.org/10.1002/adfm.200901363.

[12] Khan, M. S., Fon, D., Li, X., Tian, J., Forsythe, J., Garnier, G., \& Shen, W. (2010). Biosurface engineering through ink jet printing. Colloids and Surfaces B: Biointerfaces, 75(2), 441-447. https://doi.org/10.1016/j.colsurfb.2009.09.032.

[13] Gimenez, A. J., Luna-Barcenas, G., Sanchez, I. C., \& Yanez-Limon, J. M. (2015). Paper-based ZnO oxygen sensor. IEEE Sensors Journal, 15(2), 12461251. https://doi.org/10.1109/JSEN.2014.2361780. 